\title{
VARIAÇÃO RADIAL DA RETRATIBILIDADE E DENSIDADE BÁSICA DA MADEIRA DE Eucalyptus saligna $\mathrm{Sm} .{ }^{1}$
}

\author{
José Tarcísio da Silva Oliveira² e José de Castro Silva ${ }^{3}$
}

\begin{abstract}
RESUMO - A ultra-estrutura e a composição química da madeira, bem como suas propriedades físicas e mecânicas, variam significativamente entre espécies, entre árvores de uma mesma espécie e, mesmo, entre diferentes partes de uma mesma árvore. Com este trabalho objetivou-se o estudo dos parâmetros de retratibilidade e de densidade básica da madeira Eucalyptus saligna, com idade de 16 anos, proveniente de talhões experimentais da EMBRAPA Florestas, de Colombo, Paraná. As amostras foram retiradas à altura do DAP de quatro posições eqüidistantes a partir da medula em direção à periferia, correspondendo a 0,33 , 66 e $100 \%$, com dimensões nominais de $1,0 \times 2,0$ x 3,0 cm, sendo a última dimensão no sentido longitudinal. Elas foram mantidas em câmara fechada com ventilação, próximo de soluções salinas supersaturadas, com o objetivo de proporcionar diferentes condições de umidade relativa. Uma vez atingidas as distintas condições de umidade de equilíbrio, as amostras foram secas em estufa a $105^{\circ} \mathrm{C}$ e obtidos os dados de retratibilidade e densidade básica da madeira nas posições mencionadas. Constataramse valores de contração volumétrica mais baixos na região medular, apresentando um acréscimo para as demais posições. Comportamento semelhante foi observado para os coeficientes das contrações lineares nas direções tangencial e radial. O fator anisotrópico foi consideravelmente mais elevado na região medular, decrescendo substancialmente em direção ao alburno. A densidade básica não mostrou sinais efetivos de estabilidade, apesar de mostrar tendência de aumento em direção à periferia do tronco.
\end{abstract}

Palavras-chave: Retratibilidade, Eucalyptus saligna, densidade básica e variação radial.

\section{RADIAL VARIATION OF SHRINKAGE AND SPECIFIC GRAVITY IN Eucalyptus saligna Sm. WOOD}

\begin{abstract}
The ultra structure and chemical composition of wood, as well as its physical and mechanical properties vary significantly among species, trees of a given species and even among different parts of a given tree. This work aimed to study the parameters of shrinkage and basic density of 16-year-old Eucalyptus saligna wood, originated from Embrapa Forests experimental stands in Colombo, Parana. The samples were collected at $D B H$ from four equidistant positions from the pith to the bark, corresponding to 0,33,66 and 100\%, with nominal dimensions of $1.0 \times 2.0 \times 3.0 \mathrm{~cm}$, with the latter being longitudinal-wise. They were kept inside a closed chamber under ventilation and near supersaturated saline solutions so as to provide different relative humidity conditions. Once these conditions were reached, the samples were oven-dried at $105{ }^{\circ} \mathrm{C}$. Shrinkage and basic density data were obtained in the four populations studied. Lower volumetric shrinkage values were found near the pith, increasing in the other positions. Similar behavior was observed for the linear shrinkage coefficients in the tangential and radial directions. The anisotropic factor, i.e., the relationship between tangential and radial shrinkage (T/R), was highest in the pith region, decreasing substantially towards the sapwood. Basic density did not show any signs of effective stability, despite its tendency to increase towards the outer stem.
\end{abstract}

Key words: $\quad$ Shrinkage, Eucalyptus saligna, basic density and radial variation.

1 Recebido para publicação em 18.9.2001.

Aceito para publicação em 12.5.2003.

2 Departamento de Engenharia Rural da Universidade Federal do Espírito Santo - UFES, Caixa Postal 16, $29500-000$ AlegreES. ${ }^{3}$ Dep. de Engenharia Florestal da Universidade Federal de Viçosa - UFV, 36571-000 Viçosa-MG. 


\section{INTRODUÇÃO}

A madeira é originária de um sistema biológico complexo, tornando-se um material de extrema variabilidade. Sua ultra-estrutura e composição química, bem como suas propriedades físicas e mecânicas, variam significativamente entre espécies, entre árvores de uma mesma espécie e, mesmo, entre diferentes partes de uma mesma árvore. A variabilidade, geralmente encontrada dentro de uma árvore individual, deve-se, provavelmente, às mudanças sofridas pelo câmbio durante o envelhecimento e modificações impostas pelas condições ambientais.

Todo material higroscópico, como a madeira e vários outros materiais celulósicos, apresenta contração quando o seu teor de umidade do ponto de saturação das fibras (psf) é reduzido até à condição absolutamente seca ou anidra. A contração e a expansão higroscópica da madeira são dois dos mais importantes problemas práticos que ocorrem durante a sua utilização, como conseqüência da mudança do teor de umidade. A magnitude das variações dimensionais depende de inúmeros fatores, como o teor de umidade, a direção estrutural (radial, tangencial ou longitudinal), a posição dentro da árvore, a densidade da madeira, a temperatura, o grau de estresse de secagem causada pelo gradiente de umidade, entre outros.

Devido à característica anisotrópica, as mudanças dimensionais observadas na madeira são diferentes ao longo das três direções estruturais. Em geral, a contração na direção tangencial é, aproximadamente, duas vezes maior do que na direção radial; a razão entre a contração tangencial e radial (relação T/R), comumente chamada de fator de anisotropia, geralmente varia de 1,5 a 2,5, e tornou-se um índice muito importante nos estudos de contração de madeira; quanto maior essa relação, maior será a tendência ao fendilhamento e empenamento da madeira. Para usos que envolverem estabilidade dimensional da madeira, a mais recomendada é aquela que apresentar a menor taxa $\mathrm{T} / \mathrm{R}$.

Segundo Panshin \& De Zeeuw (1980), a contração da madeira varia com a sua posição na árvore. Normalmente, as contrações transversais são menores próximo à medula (madeira juvenil), crescendo rapidamente desta para a casca, estando essa mudança relacionada com a rápida redução do ângulo microfibrilar na parede celular, com o aumento do comprimento da célula e do teor de celulose. Oliveira (1998), estudando várias espécies de eucalipto, verificou um aumento na retratibilidade volumétrica total, com o aumento radial a partir da

R. Árvore, Viçosa-MG, v.27, n.3, p.381-385, 2003 medula. Hillis \& Brown (1978) afirmaram que a madeira proveniente de árvores do gênero Eucalyptus, de rápido crescimento, apresenta uma contração excessiva e ocasiona defeitos de secagem, como empenamentos e fendilhamentos, que tendem a ser piores em madeiras de menor densidade. Os autores relataram que tais defeitos são mais severos na zona ao redor da medula.

As variações da densidade dependem das mudanças na proporção dos vasos e das espessuras das paredes celulares das fibras. O crescimento da densidade pode ser o resultado do aumento da espessura da parede celular das fibras ou de um aumento na proporção das fibras em relação, por exemplo, à proporção de vasos. De maneira inversa, um aumento na proporção de vasos, com ou sem decréscimo na espessura da parede celular, leva à redução na densidade. Segundo Panshin \& De Zeeuw (1980), os efeitos são interativos e difíceis de ser avaliados isoladamente.

O objetivo do presente trabalho foi estudar os parâmetros de retratibilidade e de densidade básica da madeira de Eucalyptus saligna, em quatro posições eqüidistantes a partir da medula em direção à periferia, na altura do DAP.

\section{MATERIAL E MÉTODOS}

A madeira utilizada no ensaio experimental foi proveniente de árvores de Eucalyptus saligna Sm., com idade de 16 anos, procedente de talhões experimentais da EMBRAPA Florestas, de Colombo, Paraná. Os ensaios foram realizados no Laboratório de Propriedades Físicas e Mecânicas da Madeira do Departamento de Engenharia Florestal da Universidade Federal de Viçosa. Utilizaramse cinco árvores, cuja amostragem foi feita na região do DAP, onde foram tomadas quatro posições eqüidistantes na direção medula-casca, correspondendo a $0,33,66$ e $100 \%$, com 20 repetições por posição, totalizando 400 amostras.

As amostras de formato retangulares apresentaram dimensões de 1,0 × 2,0 × 3,0 cm, sendo a última medida no sentido longitudinal. Inicialmente em estado verde, as amostras foram pesadas em balança eletrônica digital com precisão de $0,01 \mathrm{~g}$, medidas linearmente com um pálmer de precisão $0,001 \mathrm{~mm}$, diretamente sobre as amostras, e determinado o volume em mercúrio pelo método da balança hidrostática. Após a secagem natural ao ar, as amostras foram colocadas em estufa a $103 \pm 2{ }^{\circ} \mathrm{C}$, para tomadas da massa, das dimensões e do volume, na 
condição anidra. Finalizada a coleta dos dados, procederam-se aos cálculos de retratibilidade e densidade básica, bem como a análise estatística e confecções de gráficos ilustrativos da variação das propriedades na direção radial.

\section{RESULTADOS E DISCUSSÃO}

Os resultados dos coeficientes de retratibilidade total e densidade básica para a madeira de Eucalyptus saligna, em quatro posições eqüidistantes a partir da medula, estão apresentados no Quadro 1.

No Quadro 1, observou-se que os valores de contração volumétrica são crescentes no sentido medulacasca, à exceção da posição $3(66 \%)$, região correspondente ao cerne periférico. Comportamento semelhante foi também observado para as contrações lineares nas direções tangencial e radial das amostras. Constatou-se uma diferença relativamente acentuada entre a contração volumétrica apresentada e o somatório das respectivas contrações lineares. Tal diferença se deve à utilização de diferentes metodologias para obtenção das contrações, considerando que a contração volumétrica foi obtida pelo método da balança hidrostática e as contrações lineares foram obtidas através de medição direta nas amostras, com o pálmer. Ela também pode ser atribuída a um possível colapso ocorrido em determinadas amostras, acarretando uma dificuldade na medição correta das retratibilidades lineares. Com relação ao fator anisotrópico, observou-se que nas duas posições iniciais a partir da medula este índice foi superior ao valor médio para esta madeira, igual a 1,99. Nas posições mais periféricas do fuste, observaram-se valores inferiores ao valor médio; a região periférica se destacou por apresentar o menor índice e, conseqüentemente, uma madeira de maior estabilidade dimensional, em função da menor variabilidade entre as contrações tangencial e radial.

Oliveira (1997), estudando várias espécies de eucalipto, verificou um padrão crescente de retratibilidade na direção medula-casca. Quanto à densidade básica, observaram-se valores muito baixos na região medular, com tendência de crescimento nas posições mais externas do tronco. Constatou-se que para esta propriedade o valor médio somente foi ultrapassado nas duas posições mais periféricas do xilema secundário. Os elevados valores dos coeficientes de variação e desvio-padrão para os coeficientes e a retratibilidade indicam a produção de lenhos bastante variáveis no que diz respeito a estas importantes propriedades. Em relação à densidade básica, observaram-se valores de coeficiente de variação e desvio-padrão bastante satisfatórios, apesar de sua destacada variação na direção radial. A variação dos coeficientes de contração na direção radial para a madeira em estudo pode ser mais bem visualizada através da Figura 1.

Quadro 1 - Valores médios para os coeficientes de retratibilidade e densidadebásica da madeira Eucalyptus saligna, tomados de quatro posições eqüidistantes a partir da medula em direção à casca

Table 1 - Average of the shrinkage coefficient and specific gravity values to four halfway positions from the pith to the bark of Eucalyptus saligna wood

\begin{tabular}{|c|c|c|c|c|c|c|}
\hline \multirow{2}{*}{$\begin{array}{c}\text { Posição } \\
\text { Medula-Casca }\end{array}$} & \multirow{2}{*}{$\mathrm{N}^{*}$} & \multicolumn{3}{|c|}{ Contrações (\%) } & \multirow{2}{*}{$\begin{array}{c}\text { Relação } \\
\text { T/R }\end{array}$} & \multirow{2}{*}{$\begin{array}{c}\text { Densidade } \\
\text { Básica }\left(\mathrm{g} / \mathrm{cm}^{3}\right)\end{array}$} \\
\hline & & Volumétrica & Tangencial & Radial & & \\
\hline 1 & 74 & $\begin{array}{c}21,28^{\underline{1 /}} \\
(25,7)(5,47)\end{array}$ & $\begin{array}{c}10,89 \\
(19,3)(2,10)\end{array}$ & $\begin{array}{c}4,99 \\
(19,5)(0,97)\end{array}$ & $\begin{array}{c}2,22 \\
(19,2)(0,43)\end{array}$ & $\begin{array}{c}0,39 \\
(9,6)(0,04)\end{array}$ \\
\hline 2 & 83 & $\begin{array}{c}27,18 \\
(24,5)(6,65)\end{array}$ & $\begin{array}{c}15,20 \\
(18,5)(2,81)\end{array}$ & $\begin{array}{c}7,06 \\
(19,5)(1,37)\end{array}$ & $\begin{array}{c}2,18 \\
(14,9)(0,32)\end{array}$ & $\begin{array}{c}0,43 \\
(11,5)(0,05)\end{array}$ \\
\hline 3 & 93 & $\begin{array}{c}26,57 \\
(20,6)(5,48)\end{array}$ & $\begin{array}{c}15,67 \\
(18,5)(2,90)\end{array}$ & $\begin{array}{c}8,59 \\
(19,8)(1,70)\end{array}$ & $\begin{array}{c}1,84 \\
(13,4)(0,25)\end{array}$ & $\begin{array}{c}0,50 \\
(6,5)(0,03)\end{array}$ \\
\hline 4 & 90 & $\begin{array}{c}28,69 \\
(26,2)(7,52)\end{array}$ & $\begin{array}{c}16,88 \\
(25,6)(4,32)\end{array}$ & $\begin{array}{c}9,47 \\
(23,9)(2,26)\end{array}$ & $\begin{array}{c}1,79 \\
(12,0)(0,21)\end{array}$ & $\begin{array}{c}0,55 \\
(7,8)(0,04)\end{array}$ \\
\hline Média Geral & 340 & $\begin{array}{c}26,00 \\
(26,5)(6,9)\end{array}$ & $\begin{array}{c}14,83 \\
(25,9)(3,85)\end{array}$ & $\begin{array}{c}7,67 \\
(30,7)(2,35)\end{array}$ & $\begin{array}{c}1,99 \\
(18,1)(0,36)\end{array}$ & $\begin{array}{c}0,47 \\
(15,6)(0,07)\end{array}$ \\
\hline
\end{tabular}

* número de repetições. ${ }^{1 /}$ Valores entre parêntesis são coeficiente de variação e desvio-padrão. 
Na Figura 1, podem ser observados valores inferiores dos coeficientes de contração volumétrica e lineares na região medular. Tais valores podem ser o reflexo de grandes ângulos microfibrilares, que são comuns nas regiões mais internas do tronco, acarretando, conseqüentemente, maiores contrações longitudinais e menores coeficientes de contrações na direção transversal. Em E. microcorys, Lima (1996) observou tendência de aumento de todos os coeficientes de contração examinados no sentido medula-casca, com uma pequena queda, às vezes, na madeira do alburno. Em E. pilularis, o autor afirmou que, a despeito da grande flutuação nos valores, houve tendência de as contrações serem menores no alburno. Para as duas espécies, o fator anisotrópico decresceu no sentido medula-casca.

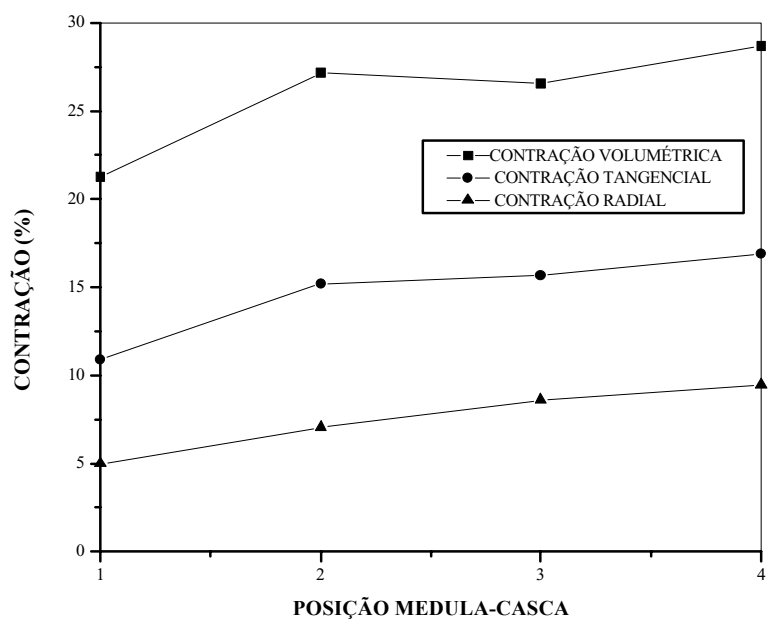

Figura 1 - Representação gráfica da variação das contrações volumétrica e lineares na direção medula-casca para a madeira de Eucalyptus saligna.

Figure 1 - Variation of the transverse and volumetric shrinkage from the pith to the bark in Eucalyptus saligna wood.

A Figura 2 ilustra graficamente a variação do fator anisotrópico, na direção radial para a madeira de E. saligna. O gráfico da Figura 2 destaca muita bem a existência de uma madeira mais instável dimensionalmente na região medular, que deverá sofrer maior empenamento e, principalmente, fendilhamento quando sujeita a diferentes condições de umidade relativa.

Por outro lado, observaram-se valores reduzidos desse fator na região do alburno. Pode-se inferir que a região mais externa destes fustes irá produzir peças de maior estabilidade dimensional e, conseqüentemente, menos sujeitas a empenamentos e fendilhamentos durante a fase de secagem. Oliveira (1998), estudando sete espécies de madeira de eucalipto, observou tendência geral para valores de $T / R$ inferiores na região do alburno, indicando, assim, elevada estabilidade dimensional.

A variação da densidade básica da madeira de E. saligna é mais bem visualizada na Figura 3 .

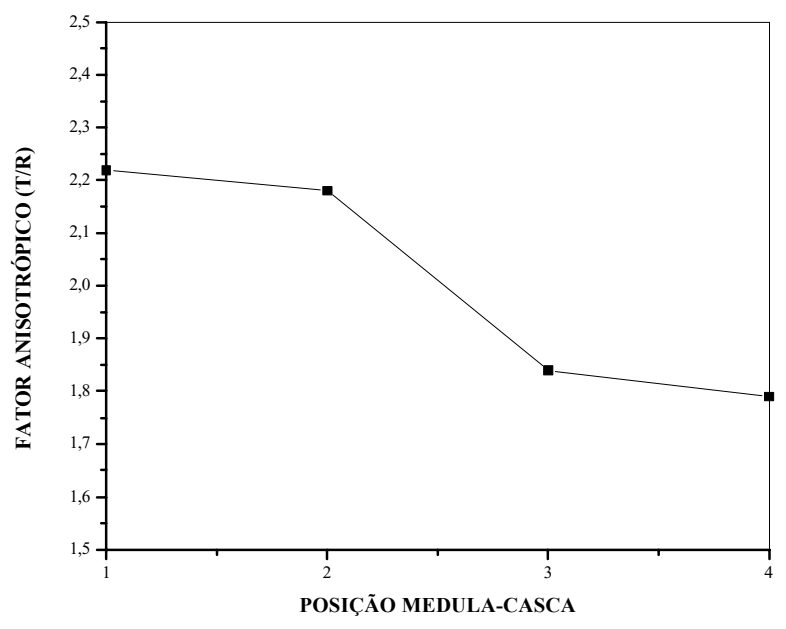

Figura 2 - Representação gráfica da variação do fator anisotrópico (T/R) total na direção medula-casca para madeira de Eucalyptus saligna.

Figure 2 -Variation of the anisotropic factor from the pith to the bark in Eucalyptus saligna wood.

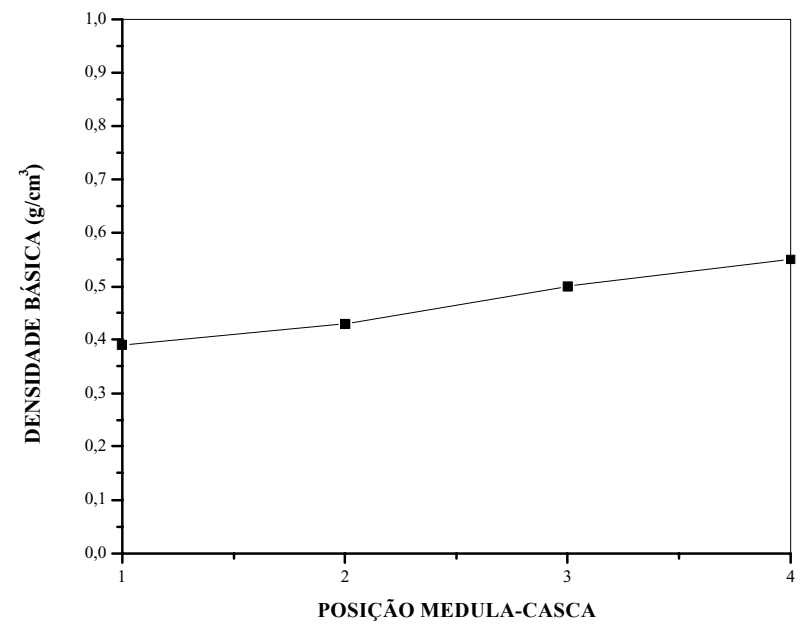

Figura 3 - Representação gráfica da variação da densidade básica da madeira de Eucalyptus saligna na direção medula-casca.

Figure 3 - Variation of the specific gravity in the wood of Eucalyptus saligna from the pith to the bark. 
Na Figura 3, observou-se o perfil crescente de densidade básica na direção medula-casca para a madeira de eucalipto em estudo. Através do gráfico constatou-se uma pequena variação nos valores de densidade das quatro posições estudadas. Acredita-se que a madeira formada nas duas primeiras posições destes fustes é somente constituída de material juvenil. Oliveira (1998), estudando a densidade básica da madeira proveniente de sete espécies de eucalipto, observou que apesar do comportamento geral de aumento na direção medula-casca cada espécie apresentou um perfil específico de variação, inclusive com variantes para cada altura do tronco. Carmo (1996) estudou as variações da densidade básica, do teor de umidade, dos coeficientes de contração e dos teores de extrativos no sentido medula-casca e ao longo do tronco de três árvores de seis espécies de eucalipto, ou seja: Eucalyptus grandis, E. saligna, E. citriodora, E. pilularis, E. cloeziana e E. maculata. A autora verificou que em todas as espécies houve um aumento da densidade básica no sentido medula-casca e no sentido da altura, com valores mínimos nas proximidades da medula e máximas na região do cerne periférico ou no alburno.

\section{CONCLUSÕES}

Pelos resultados apresentados e discutidos anteriormente, conclui-se que:

- A retratibilidade varia na direção radial da madeira de Eucalyptus saligna, ocorrendo valores menores na região da medula com acréscimo até a região mais periférica do tronco.

- O fator anisotrópico é consideravelmente mais elevado na região medular, caindo substancialmente em direção ao alburno da madeira de E. saligna.
- A densidade básica, apesar de mostrar tendência de aumento em direção à periferia do tronco, ainda não mostrou sinais efetivos de estabilidade, o que leva a supor uma grande presença de madeira juvenil.

\section{AGRADECIMENTO}

Os autores agradecem ao Dr. José Carlos Duarte Pereira e ao Centro Nacional de Pesquisas Florestais da EMBRAPA - Colombo-PR, pela cessão da madeira utilizada e pelas informações dispensadas para realização desse trabalho.

\section{REFERÊNCIAS BIBLIOGRÁFICAS}

CARMO, A. P. T. Avaliação de algumas propriedades da madeira de seis espécies de eucalipto. 1996. $74 \mathrm{f}$.

Dissertação (Mestrado em Ciência Florestal) - Universidade Federal Federal de Viçosa, Viçosa, 1996.

HILLIS, W. E.; BROWN, A. G. (Eds.) Eucalypts for wood production. Melbourne: CSIRO, 1978.

LIMA, T. G. Variações no sentido radial e longitudinal de algumas propriedades das madeiras de Eucalyptus microcorys F. Muell e Eucalyptus pilularis Sm. 1996. 106 f. Dissertação (Mestrado em Ciência Florestal) Universidade Federal de Viçosa, Viçosa, 1996.

OLIVEIRA, J. T. S. Caracterização da madeira de eucalipto para a construção civil. 1998. $429 \mathrm{f}$. Tese (Doutorado) - Escola Politécnica, Universidade de São Paulo, São Paulo, 1998.

PANSHIN, A. J.; DE ZEEUW, C. Textbook of wood technology. 4.ed. New York: Mc-Graw-Hill, 1980. 